\title{
A simple approximation algorithm for WIS based on the approximability in $k$-partite graphs
}

\author{
Jérôme Monnot*
}

\begin{abstract}
Résumé
Dans cette note, nous montrons comment une solution optimale ou une solution approchée du stable pondéré dans les graphes $k$-partis peut permettre de récupérer une bonne solution approchée du stable pondéré dans les graphes généraux. Plus précisément, un stable pondéré optimal dans les graphes bipartis nous permet d'obtenir une $\frac{2}{\Delta(G)}$-approximation et, plus généralement, une $\rho$ solution approchée dans les graphes $k$-partis fournit une $\frac{k}{\Delta(G)} \rho$ solution approchée dans les graphes généraux.
\end{abstract}

Mots-clefs : Algorithme approché, coloration, stable pondéré, graphe $k$-parti.

\begin{abstract}
In this note, we show how optimal or approximate weighted independent sets in $k$-partite graphs may yield to a good approximate weighted independent set in general graphs. Precisely, optimal solutions in bipartite graphs do yield to a $\frac{2}{\Delta(G)}{ }^{-}$ approximation and, more generally, $\rho$-approximate solutions in $k$-partite graphs yield to a $\frac{k}{\Delta(G)} \rho$-approximation in general graphs.
\end{abstract}

Key words : Approximation algorithms, Coloring, Weighted Independent Set, $k$ partite graphs.

* LAMSADE, Université Paris-Dauphine, 75775 Paris cedex 16, France. \{monnot\} @lamsade.dauphine.fr 


\section{Introduction}

In the Maximum Weighted Independent Set problem (WIS, for short), we are given a simple, connected, undirected and loop-free graph $G=(V, E)$ on $n$ vertices, with maximum degree $\Delta(G)$. Each vertex $v$ in $V$ is labelled with a positive weight $w(v) \geq 0$. For a subset $S \subseteq V$ of vertices, we denote by $w(S)=\sum_{v \in S} w(v)$ the sum of the weights of the elements in $S$. The goal of WIS is to find an independent set $S$ (that is a subset of pairwise non-adjacent vertices) in $G$ that maximizes $w(S)$. When the weight of each vertex is equal to one, this problem is usually called Maximum Independent Set problem (IS, for short).

WIS is known to be NP-hard in general graphs, but also for certain classes of graphs (see Garey and Johnson [5]). On the other hand, for a variety of graphs from both practical and theoretical frameworks, which includes the Perfect graphs family, this problem is polynomial, Grotschel et al. [6]. Very recently, Alekseev and Lozin, [1] provided a complete characterization of the $(p, q)$-colorable graphs for which WIS is polynomial, where we recall that a graph is $(p, q)$-colorable if it can be partitioned into at most $p$ cliques and $q$ independent sets. The main result states that WIS is polynomial on $(p, q)$-colorable graphs if and only if $q \leq 2$ (under the assumption $\mathbf{P} \neq \mathbf{N P}$ ). In particular, since a bipartite graph is a perfect graph as well as a $(0,2)$-colorable graph, then the Maximum Weighted Independent Set problem is polynomial on bipartite graphs.

Because WIS is one of the most important problem from both a practical and a theoretical point of view, many approximation results have been found out by several authors (see, for instance Hochbaum [9], Halldórsson and Lau [7] for performance ratio only depending on $\Delta(G)$, Halldórsson [8], Halldórsson and Lau [7] and Demange and Paschos [4]). Very recently, Sakai et al. [13] studied the behavior of several greedy strategies on WIS. In particular, they proved that one of these greedy algorithms is a $\frac{1}{\Delta(G)}$ approximation and that this ratio is tight. This algorithm selects, as long as the current graph $G_{i}$ is not empty, a vertex $v$ maximizing $\frac{w(v)}{d_{G_{i}}(v)+1}$ (where $d_{G_{i}}(v)$ is the degree of $v$ in the current graph $G_{i}$ ), and then deletes $v$ and its neighborhood from the current graph.

Results of this paper. In this paper, we show how an optimum weighted independent set in bipartite graphs and a $\rho$-approximation of WIS in $k$-partite graphs respectively allows to obtain a $\frac{2}{\Delta(G)}$-approximation and a $\frac{k}{\Delta(G)} \rho$-approximation in general graphs. In order to build a $k$-partite graph from a given graph, we use the notion of coloring, that is a partition of the vertices into independent sets (see, Paschos [12], for a survey on the approximability of the coloring problem). Already in the past, Hochbaum [9], exploits this notion of coloring to obtain a $\frac{2}{\Delta(G)}$-approximation of WIS, but in a complete different way. The algorithm of [9] is based on a preprocessing due to Nemhauser and Trotter [11] which provides two disjoint subgraphs, including an independent set; it then computes 
on the other subgraph a coloring from which it selects the best independent set which is finally added to the first subgraph. More recently, Halldórsson and Lau [7] proposed an elegant algorithm which consists in partitioning $G$ into at most $\left\lceil\frac{\Delta(G)+1}{3}\right\rceil$ subgraphs $G_{i}$ of degree at most 2 ; then, for each $G_{i}$, an optimum weighted independent set in $G_{i}$ is computed and finally, the best of these solutions is returned. The performance ratio of this algorithm is $1 /\lceil(\Delta(G)+1) / 3\rceil$, that is better that $2 / \Delta(G)$ as soon as $\Delta(G) \geq 7$ or $\Delta(G)=5$. Our algorithm computes, for every $k$-partite graph built on the coloring, an optimum or an approximate solution; it then returns the best one as a solution of the initial problem.

\section{The Algorithm}

In the first algorithm, we only use the notion of bipartite graph.

\section{Algorithm 1}

1 Find a coloring $\mathcal{S}=\left(S_{1}, \ldots, S_{\ell}\right)$ by using a polynomial-time algorithm $A$;

2 For any $1 \leq i<j \leq \ell$ do

2.1 Find an optimal independent set $S_{i, j}$ of bipartite graph induced by $S_{i} \cup S_{j}$;

3 Return $S=\operatorname{argmax}\left\{w\left(S_{i, j}\right): 1 \leq i<j \leq \ell\right\}$;

This algorithm is trivially polynomial since we apply at most $O\left(\ell^{2}\right)$ times a polynomial procedure.

Theorem 2.1 Algorithm 1 is a $\frac{2}{\ell}$-approximation for WIS in general graphs.

Proof. Let $I=(G, w)$ be an instance of WIS and let $S^{*}$ be an optimal solution with value $\operatorname{opt}(I)=w\left(S^{*}\right)$. We set $S_{i}^{*}=S^{*} \cap S_{i}$ for $i \leq \ell$ where $\mathcal{S}=\left(S_{1}, \ldots, S_{\ell}\right)$ is the coloring provided by algorithm $A$.

For any $i, j$ with $j>i$, the following key result holds:

$$
w(S) \geq w\left(S_{i}^{*}\right)+w\left(S_{j}^{*}\right)
$$

Let us explain why this result is true: on the one hand, the set $S_{i}^{*} \cup S_{j}^{*}$ is an independent set of the bipartite graph induced by $S_{i} \cup S_{j}$ and, on the other hand, by construction of the 
algorithm, $S_{i, j}$ is an optimal solution of this bipartite graph. Thus, since $w(S) \geq w\left(S_{i, j}\right)$, we deduce the expected result.

Summing the inequalities (1) for $i=1$ to $i=\ell-1$ and $j=i+1$ to $j=\ell$, we obtain:

$$
\begin{aligned}
\sum_{i=1}^{\ell-1} \sum_{j=i+1}^{\ell} w(S) & \geq \sum_{i=1}^{\ell-1} \sum_{j=i+1}^{\ell}\left(w\left(S_{i}^{*}\right)+w\left(S_{j}^{*}\right)\right) \\
& \geq \sum_{i=1}^{\ell-1}(\ell-i) w\left(S_{i}^{*}\right)+\sum_{i=1}^{\ell-1} \sum_{j=i+1}^{\ell} w\left(S_{j}^{*}\right) \\
& \geq \sum_{i=1}^{\ell-1}(\ell-i) w\left(S_{i}^{*}\right)+\sum_{j=2}^{\ell} \sum_{i=1}^{j-1} w\left(S_{j}^{*}\right) \\
& \geq \sum_{i=1}^{\ell-1}(\ell-i) w\left(S_{i}^{*}\right)+\sum_{j=2}^{\ell}(j-1) w\left(S_{j}^{*}\right) \\
& \geq(\ell-1) w\left(S_{1}^{*}\right)+\sum_{i=2}^{\ell-1}(\ell-i) w\left(S_{i}^{*}\right)+\sum_{i=2}^{\ell-1}(i-1) w\left(S_{i}^{*}\right)+(\ell-1) w\left(S_{\ell}^{*}\right) \\
& \geq(\ell-1) \sum_{i=1}^{\ell} w\left(S_{i}^{*}\right)
\end{aligned}
$$

Thus, since $\sum_{i=1}^{\ell} w\left(S_{i}^{*}\right)=w\left(S^{*}\right)$, and $\sum_{i=1}^{\ell-1} \sum_{j=i+1}^{\ell} w(S)=\frac{\ell(\ell-1)}{2} w(S)$, we obtain:

$$
\frac{\ell(\ell-1)}{2} w(S) \geq(\ell-1) \operatorname{opt}(I)
$$

and the result follows.

We know that we can easily obtain a coloring using at most $\Delta(G)+1$ colors (see Berge [2]): starting from an arbitrary coloring, pick up each vertex $v$ with a color at least $\Delta(G)+2$ and recolor it with a compatible color among $\{1, \ldots, \Delta(G)+1\}$ (this is always possible, since $v$ has at most $\Delta(G)$ neighbors). Thus, using Theorem 2.1 and the inequality $\ell \leq \Delta(G)+1$, we deduce:

Corollary 2.2 WIS is $\frac{2}{\Delta(G)+1}$-approximable and this ratio is tight.

Proof. We show that this ratio is tight, even in the basis case of the maximum independent set problem (i.e., $\forall v \in V, w(v)=1$ ). We consider a graph $G=(V, E)$ on $2 n(\Delta+1)$ vertices which are partitioned into a coloring $\mathcal{S}=\left(S_{1}, \ldots, S_{\Delta+1}\right)$ where $S_{i}=$ $\left\{v_{i, 1}, \ldots, v_{i, 2 n}\right\}$. Moreover, there is an edge between every couple of vertices $\left(v_{i, k}, v_{j, n+k}\right)$ and $\left(v_{i, n+k}, v_{j, k}\right)$ where $j \neq i$ and $k=1, \ldots, n$.

Observe that $G$ is $\Delta$-regular. Assume that the algorithm $A$ produces the coloring $\mathcal{S}=$ $\left(S_{1}, \ldots, S_{\Delta+1}\right)$; then, the Algorithm 1 returns $S_{1}$ with a size $2 n$ whereas an optimal solution is given by the set $\left\{v_{i, j}: 1 \leq i \leq \Delta+1,1 \leq j \leq n\right\}$ with a size $(\Delta+1) n$.

Remark that this instance also allows us to prove that even a more sophisticated algorithm has no better performance. In this algorithm, we first apply Algorithm 1 and then compute the best weighted independent set on every bipartite graph induced 
by $S_{i_{1}, j_{1}} \cup S_{i_{2}, j_{2}}$, where the sets $S_{i_{1}, j_{1}}$ and $S_{i_{2}, j_{2}}$ describe the optimal independent sets produced by Algorithm 1.

We can slightly improve this bound to $\frac{2}{\Delta(G)}$, by using the Brook's theorem, [3] and the constructive proof of Lovasz [10].

Corollary 2.3 WIS is $\frac{2}{\Delta(G)}$-approximable, when $G$ is $K_{\Delta(G)+1}$-free.

One way to improve this bound is to consider $k$-partite graphs instead of bipartite graphs, where $k$ is a universal constant. Thus, the new algorithm consists in modifying the step 2.1 by finding an optimal independent set $S_{i_{1}, \ldots, i_{k}}$ on the $k$-partite graph induced by $S_{i_{1}} \cup \ldots \cup S_{i_{k}}$ for each $i_{1}<\ldots<i_{k}$. Some algebra shows that this algorithm is a $\frac{k}{\ell}$-approximation for WIS. Unfortunately, such an algorithm does not run in polynomial time (even for $k=3$ ), since computing an optimal independent set in $k$-partite graphs is NP-hard (see the characterization of the complexity of WIS in $(p, q)$-colorable graphs in [1]). In those circumstances, we replace an optimal solution by an approximate solution, and the whole algorithm writes now:

Algorithm 2

1 Find a coloring $\mathcal{S}=\left(S_{1}, \ldots, S_{\ell}\right)$ by using a polynomial-time algorithm $A$;

2 For any $1 \leq i_{1}<\ldots<i_{k} \leq \ell$ do

2.1 Find an approximate independent set $S_{i_{1}, \ldots, i_{k}}$ of the $k$-partite graph induced by $S_{i_{1}} \cup \ldots \cup S_{i_{k}}$ using an algorithm $B$;

3 Return $S=\operatorname{argmax}\left\{w\left(S_{i_{1}, \ldots, i_{k}}\right): 1 \leq i_{1}<\ldots<i_{k} \leq \ell\right\}$

This algorithm is polynomial as soon as the algorithm $B$ runs in polynomial time ( $k$ being a constant not depending on the instance size).

Theorem 2.4 If algorithm $B$ is a $\rho$-approximation of WIS in k-partite graphs, then Algorithm 2 is an $\frac{k}{\ell} \rho$-approximation for WIS in general graphs.

Proof. Let $B$ be an algorithm which yields to a $\rho$-approximation of WIS in $k$-partite graphs, and let $S^{*}$ be an optimal solution on a given graph $G$. As previously, we set $S_{i}^{*}=S^{*} \cap S_{i}$ for $i \leq \ell$ where $\mathcal{S}=\left(S_{1}, \ldots, S_{\ell}\right)$ is the coloring provided by the algorithm $A$. 
The inequality 1 becomes: for any $i_{1}, \ldots, i_{k}$ with $i_{k}>\ldots>i_{1}$, we have

$$
w(S) \geq \rho \sum_{j=1}^{k} w\left(S_{i_{j}}^{*}\right)
$$

In order to see that, just remark that $S_{i_{1}}^{*} \cup \ldots \cup S_{i_{k}}^{*}$ is a feasible independent set on the $k$-partite graph induced by $S_{i_{1}} \cup \ldots \cup S_{i_{k}}$ (we denoted by $G^{\prime}$, this graph); since $S_{i_{1}, \ldots, i_{k}}$ is a $\rho$-approximation on $G^{\prime}$, we get $w\left(S_{i_{1}, \ldots, i_{k}}\right) \geq \rho \operatorname{opt}\left(G^{\prime}\right) \geq \rho\left(w\left(S_{i_{1}}^{*}\right)+\ldots+w\left(S_{i_{k}}^{*}\right)\right)$.

Summing up inequalities (3) for all $i_{1}, \ldots, i_{k}$ such that $1 \leq i_{1}<\ldots<i_{k} \leq \ell$, we obtain:

$$
\frac{\ell(\ell-1) \ldots(\ell-k+1)}{k(k-1) \ldots 2} w(S) \geq \rho \sum_{i=1}^{\ell} \frac{(\ell-1) \ldots(\ell-k+1)}{(k-1) \ldots 2} w\left(S_{i}^{*}\right)
$$

Actually, when summing the inequalities (3), the term $w(S)$ appears exactly as many times as the number of choices of $k$ elements among $\ell$, and each $w\left(S_{i}^{*}\right)$ appears as many times as the number of choices of $k-1$ elements among $\ell-1$. Finally, since $\sum_{i=1}^{\ell} w\left(S_{i}^{*}\right)=$ $w\left(S^{*}\right)$, the result follows.

This theorem becomes interesting when having some good bounds of the approximability of WIS in $k$-partite graphs. For instance, using Theorem 2.1, we obtain the bound $\rho=\frac{2}{3}$ for tripartite graphs; unfortunately, this does not allow to improve the bound of Theorem 2.1. Thus, we aim at strictly improving this bound of $\frac{2}{3}$ in order to improve the best performance ratio of $\frac{2}{\Delta(G)}$ or $1 /\lceil(\Delta(G)+1) / 3\rceil$ when $\Delta(G)$ is small (i.e., $\Delta(G)=3,4,6$ ).

\section{Références}

[1] V. E. AleKSEEV AND V. V. LozIN [2003]. Independent sets of maximum weight in $(p, q)$-colorable graphs. Discrete Math., (in press).

[2] C. Berge [1973]. Graphs and hypergraphs. North Holland, Amsterdam.

[3] R. L. BRooks [1941]. On colouring the nodes of a network. Proc. Cambridge Phil. Soc., 37, 194-197.

[4] M. Demange And V. Paschos [2004]. Improved approximations for weighted and unweighted graph problems. Theory of Comp. Sys., (To appear)

[5] M. R. GAREy And D. S. Johnson [1979]. Computers and intractability. A guide to the theory of NP-completeness. W. H. Freeman, San Francisco. 
[6] M. Grotschel And L. Lovasz And A. Schrijver [1984]. Polynomial algorithms for perfect graphs. Ann. Discrete Math., 21, 325-356.

[7] M. HalldóRSSON AND H. C. LAU [1997]. Low-degree graph partitioning via local search with applications to constraint satisfaction, max cut, and 3-coloring. $J$. Graph Algorithms and Applications, 1, 1-13.

[8] M. HALldóRSSON [2000]. Approximations of Weighted Independent Set and Hereditary Subset Problems. J. Graph Algorithms and Applications, 4, 1-16.

[9] D. S. Hochваum [1983]. Efficient bounds for the stable set, vertex cover and set packing problems. Discrete Applied Mathematics, 6(3), 243-254.

[10] L. LovASz [1975]. Three short proofs in graph theory. J. Combin. Theory, 19, 269271.

[11] G. L. Nemhauser AND L. E. TROTTER [1975]. Vertex packing: structural properties and algorithms. Mathematical Programming, 8, 232-248.

[12] V. Th. Paschos [2003]. Polynomial Approximation and Graph-Coloring. Сотриting, 70(1), 41-86.

[13] S. SAKai And M. Togasaki And K. YAmaZaKi [2003]. A note on greedy algorithms for the maximum weighted independent set problem. Discrete Applied Mathematics, 126, 313-322. 